\title{
Estimating Sibling Recurrence Risk in Population Sample Surveys
}

\author{
Barry I. Graubard ${ }^{\mathrm{a}}$ Monroe G. Sirken ${ }^{\mathrm{b}}$ \\ ${ }^{a}$ Division of Cancer Epidemiology and Genetics, National Cancer Institute, National Institutes of Health, \\ Bethesda, Md., and ${ }^{\mathrm{b}}$ National Center for Health Statistics, Hyattsville, Md., USA
}

\section{Key Words}

Counting rule $\cdot$ Network estimation - Sampling errors .

Diabetes

\begin{abstract}
Background/Aims: Sibling recurrence risk (SRR) is a measure of familial aggregation of a disease and is often used in family-based studies in genetic epidemiology to indicate the existence of possible genes conferring susceptibility of disease. Estimating SRR requires information about the disease status of sibships of families with two or more siblings where at least one is affected. Since family-based studies are not usually random samples, estimates of SRR derived from these studies may be biased. Network sampling used in survey research offers a way to ascertain the disease status of sibships from interviewed individuals in household survey samples, in order to obtain (approximately) unbiased estimators of SRR and its related SRR ratio (SRR divided by the prevalence of disease). Methods: Two methods of ascertaining sibships of affected families are considered: in one method the siblings' affected status is reported by an individual, regardless of the individual's affected status, and in the other method only affected individuals can report their siblings' affected status. Network estimators of SRR and SRR ratio and estimators of their standard errors are provided. Results: Reported diabetes for siblings from the 1976 National Health
\end{abstract}

Interview is used to illustrate the estimation methods. The SRR ratio for diabetes among living siblings was 5.79\% [relative standard error (RSE) $=5.12 \%$, and for living or deceased siblings, it was $7.66 \%$ (RSE $=3.76 \%$ ). Conclusions: Network sampling estimators can provide population estimates of SRR and SRR ratio for diseases such as diabetes.

Copyright $\odot 2013$ S. Karger AG, Basel

\section{Introduction}

Sibling recurrence risk (SRR) is a measure of familial aggregation that is usually of an attribute such as disease or an adverse health condition. SRR may be operationally defined as the proportion of siblings of affected individuals in a population that are affected. The measure is widely used in genetic epidemiological studies to provide evidence of possible genes conferring susceptibility to disease [1-7] and has been used to aid in the counseling

M.G.S., retired, formerly at the National Center for Health Statistics, Hyattsville, Md., USA.

An earlier less complete version of this paper was presented at the 2011 Joint Statistical Meetings: Graubard BI, Monroe SG. 2011. Estimating sibling recurrence risk in population sample surveys. In Joint Statistical Meetings Proceedings, Section on Statistics in Epidemiology. Alexandria, VA: American Statistical Association. 2623-2634.

\section{KARGER}

E-Mail karger@karger.com

www.karger.com/hhe (c) 2013 S. Karger AG, Base

0001-5652/13/0761-0018\$38.00/0
Barry I. Graubard, $\mathrm{PhD}$

Division of Cancer Epidemiology and Genetics, National Cancer Institute National Institutes of Health, 9609 Medical Center Drive, 7-E140 Bethesda, MD 20892-7234 (USA)

E-Mail graubarb@mail.nih.gov 
of patients and families about the risk of genetic diseases [8].

Typically, genetic studies that collect sibship data to estimate SRR obtain relatively small nonrandom samples, for example, from an ad hoc collection of affected individuals from clinics or physician practices. It is through the reporting by these sampled affected individuals (called probands in the genetic literature) that their sibships are ascertained along with the disease status of the members of the sibships. When the affected individuals are not a random sample, the ascertainment of sibships can be biased (ascertainment bias), resulting in potentially biased estimates of SRR derived from these samples of sibships [5]. One approach to avoid ascertainment bias is to select a simple random sample or a census of sibships from the population of sibships with at least one affected in each sibship and then estimate SRR [6]. Sampling sibships in this fashion is called complete ascertainment in the genetic literature [6]. However, population registries or sample frames of sibships from which simple random samples can be selected are often lacking. Another approach is to ascertain sibships from a simple random sample of affected individuals from the population. The sibships obtained in this fashion are assumed to be selected proportional to the number of affected living individuals in each sibship. This type of sampling of sibships is called single ascertainment. Olson and Cordell [6] provide a consistent estimator of SRR under appropriate assumptions, and variances for the estimator are given by Zou and Zhao [7]. However, because of limited population-based disease registries from which to obtain simple random samples of affected individuals, the proposed estimation of SRR derived from single ascertainment is problematic.

In this paper, we propose an alternative approach used in survey research called network sampling [9] that extends the single ascertainment approach by using large population-based household surveys to randomly sample individuals in the population (whether or not they are affected) to report on the disease status of themselves and their siblings. We allow the same sibships to be potentially reported by multiple siblings, in other words a network of siblings, but we will correct for the multiple reporting by appropriately weighting the reported sibships. This type of sampling is in contrast to conventional sampling, where each sibship is uniquely linked to one sibling, so that when this unique sibling is sampled they are the only sibling from their sibship who can report about their sibship. Conventional sampling can be considered a special case of network sampling.

Sibling Recurrence Risk in Sample Surveys
We consider two (network) estimators of SRR where each estimator is based on a different counting rule; the counting rule designates the sampled individuals through which each sibship is ascertained and the disease status of each of the siblings is determined. The first counting rule is where only affected individuals who are sampled can report the disease status of themselves and their siblings, and the second counting rule is where sampled individuals regardless of their disease status can report the disease status of themselves and their siblings. In genetic studies, affected individuals are thought to be more accurate reporters of their siblings' disease status than unaffecteds [5]. However, it was found that individuals affected with diabetes and asthma less accurately reported about the status of these diseases for their siblings than unaffected individuals [10]. These differences in reporting accuracy will be considered when we compare estimates of SRR of diabetes under the two counting rules in our data example.

In order to obtain (approximately) unbiased estimators of SRR, we weight the observations to account for (1) the differential sampling fractions used to sample individuals in the survey, and (2) the size of the sibships where larger sibships tend to have a greater probability of being reported in the survey than smaller sibships due to potential multiple reporting of a sibship according to the counting rules. Since a population survey allows also for the unbiased estimation of the population prevalence of the disease, we also consider the estimation of SRR ratio (also called sibling relative risk) which is the SRR estimate divided by an estimate of the prevalence of the disease. SRR ratio is a measure of familial aggregation of the disease relative to the prevalence of the disease and is useful for planning linkage analyses of studies of affected relative pairs [2].

This paper proposes estimators of SRR and SRR ratio and estimators of their variances from large populationbased household surveys. Household surveys use complex sample designs involving stratified multistage cluster samples. Differential sampling fractions are used to sample households and individuals from sampled households from a target population, such as the not institutionalized population of the USA. Our estimators of SRR and SRR ratio, along with their variance estimators, will be designbased to account for the complex sample design in the estimation and their variances.

In the Methodology section, we first describe the concept of a counting rule used in surveys and propose two counting rules that we later use to estimate SRR and SRR ratio of diabetes. Then, our proposed estimators of SRR 
and SRR ratio under each of our two counting rules are given, and variance estimators for our proposed SRR and SRR ratio estimators are provided. In the Results of Data Example section, the 1976 US National Health Interview Survey (NHIS) is used to illustrate our estimators for estimating family aggregation of diabetes. Even though this data set is old, it is the only publicly available national survey data set we are aware of that collected appropriate data about the disease and mortality status of sibships to allow for the estimation of family aggregation. Finally, we discuss the proposed counting rules and network estimators of SRR and SRR ratio, the collection of data needed to compute the estimators, and the results for estimating SRR and SRR ratio of diabetes from the 1976 NHIS.

\section{Methodology}

\section{Counting Rules and Network Sample Estimation}

In surveys, counting rules specify conditions for linking population elements to selection units at which the population elements are eligible to be reported in the survey [9]. In our application, the selection units are the individuals living in households in the USA in 1976 eligible to be sampled, and the population elements are the sibships of these individuals. Usually only counting rules with the property that every population element is linked to at least one selection unit are considered. This property is often needed to ensure that estimators based on the counting rule are (approximately) unbiased. In our case, this property requires each sibship to be linked to at least one individual eligible to be sampled in the survey. Two counting rules for sibship reporting are considered: (1) an All Sibling counting rule (AllSCR) where any individual who is selected in the 1976 NHIS sample can report about their sibship, and (2) an Affected Sibling counting rule (AffSCR) where only an affected individual who is selected in the 1976 NHIS sample can report about their sibship. The AffSCR violates the property stated above because sibships without any affected siblings will not be reported. However, later it will become evident that only the ascertainment of sibships with at least one affected sibling contributes to our estimators for SRR, so this violation will not result in a bias.

The selection units that are linked to each population element according to the counting rule are called the network for the population element. For example, under the AllSCR, all living brothers or sisters from the same family are the network for their sibship (throughout this paper we will only consider sibships consisting of only full siblings and not half- or adopted siblings). Since under these counting rules each sibship can be reported multiple times, where the number of possible reports for a sibship is the network size, larger sibships under the AllSCR or sibships with more affected members under the AffSCR have greater probabilities of being reported in the survey than smaller sibships or sibships with fewer siblings affected. Biased estimation of the prevalence and recurrence risk of the disease can result if the differential probabilities of reporting sibships under the sample design and counting rule are not accounted for in the estimation. A way to take account of multiple reporting in the estimation is to down-weight the ob- servations of each sibship's reports by the inverse of the number of individuals (selection units) that can report the sibship. This weight is referred to here as a network weight and is a common approach for adjusting for multiple reporting [9]. In the example of family aggregation of diabetes used in this paper, the 1976 NHIS collected reports of the number of living siblings and of the number of living diabetic siblings from each sampled individual, so that these network weights can be computed for our two counting rules.

In the next section, two estimators of SRR and SSR ratio are presented corresponding to each of the two counting rules. Because we are using counting rules with network sizes that can exceed 1 , the estimators utilizing these reports under these types of counting rules are called network estimators. To our knowledge, network estimators of SRR have not been previously investigated. Our estimators are based on the reporting of the affected status of living siblings in a family. In the Appendix, we present estimators based on the reporting of the affected status of both living and deceased siblings.

\section{Estimation of SRR and SRR Ratio}

SRR, denoted by $K_{s}$, has been formally defined in two equivalent ways [6]:

(1) The probability that a sibling of an affected individual is also affected is $P(i \in A \mid j \in A)$, where $A$ is the set of affected individuals in the population and $i$ and $j$ are a pair of siblings.

(2) The proportion of affected individuals among all siblings of affected individuals in a population.

The second definition is population-based and lends itself to being applied to population surveys. Following the notation of Zou and Zhao [7], we can express $K_{s}$ for a finite population as:

$$
K_{s}=\frac{\sum_{s=2}^{s} \sum_{a=1}^{s} a(a-1) N_{s(a)}}{\sum_{s=2}^{s} \sum_{a=1}^{s} a(s-1) N_{s(a)}},
$$

where $N_{s(a)}$ is the number of sibships in the population of size $s=$ $2, \ldots, S$, with $a$ affected siblings. Without delineating the sibship sizes, $K_{s}$ can be re-expressed as:

$$
K_{s}=\frac{\sum_{j=1}^{T} a_{j}\left(a_{j}-1\right)}{\sum_{j=1}^{T} a_{j}\left(s_{j}-1\right)},
$$

where $a_{j}$ and $s_{j}$ are the number of affected individuals in sibship $j$ and the size of sibship $j$, respectively, and $T$ is the number of sibships in the population with $a_{j} \geq 1$ and $s_{j} \geq 2$.

Suppose that in a survey $i=1, \ldots, n$ individuals are randomly sampled, and each sampled individual $i$ has a sample weight $w_{i}$, which is the inverse of the probability of including individual $i$ in the sample. A network estimator of $K_{s}$ under the AllSCR is given by:

$$
\widehat{K}_{s 1}=\frac{\sum_{i=1}^{n} \frac{w_{i}}{s_{i}} a_{i}\left(a_{i}-1\right)}{\sum_{i=1}^{n} \frac{w_{i}}{s_{i}} a_{i}\left(s_{i}-1\right)},
$$

where $a_{i}$ and $s_{i}$ are the number of affected (living) siblings and the number of (living) siblings reported by sampled individual $i$ about their sibship, respectively, and if $a_{i}=0$ or $s_{i}=1$ for all $i=1, \ldots, n$, then we set $\hat{K}_{s 1}=0$. In equ. 2 , each observation $i$ has a network weight of $1 / s_{i}$, because the network size for the sibship reported by 
sampled individual $i$ in accordance with the AllSCR is $s_{i} . \hat{K}_{s 1}$ is a (design) consistent estimator of $K_{s}$, because the expectation of the numerator and the expectation of the denominator are the numerator and denominator of $K_{s}$, respectively, where the expectations are calculated over repeated sampling from the finite population. An implicit assumption required for $\hat{K}_{s 1}$ to be a consistent estimator is that for each sibship with an affected sibling each living sibling has a positive probability of being sampled in the survey. For a US national survey such as the NHIS, this assumption requires that all living siblings live in the USA and are eligible to be sampled in the survey, i.e. are not institutionalized. In general, this assumption will only be approximately true.

Another quantity that is of interest in genetics is SRR ratio that is:

$$
\lambda_{s} \stackrel{\text { def }}{=} K_{s} / p_{a},
$$

where $p_{a}$ is the proportion or prevalence of affected individuals. Unlike most genetic studies, which obtain an estimate of $p_{a}$ from an external source, we can use the survey to obtain an estimate of $p_{a}$. An estimator of $p_{a}$ that is based on the AllSCR is:

$$
\hat{p}_{a 1}=\frac{\sum_{i=1}^{n} \frac{w_{i}}{s_{i}} a_{i}}{\sum_{i=1}^{n} w_{i}} .
$$

$\hat{p}_{a 1}$ is a network estimator that is (design) consistent and that was previously used to estimate the prevalence of diabetes; other estimators based on other counting rules can also be used to estimate $p_{a}[11]$. We combine equ. 2 and 3 to obtain a consistent network estimator of $\lambda_{s}$ as:

$$
\begin{aligned}
\hat{\lambda}_{s 1} & =\widehat{K}_{s 1} / \hat{p}_{a 1} \\
= & \frac{\sum_{i=1}^{n} \frac{w_{i}}{s_{i}} a_{i}\left(a_{i}-1\right) \sum_{i=1}^{n} w_{i}}{\sum_{i=1}^{n} \frac{w_{i}}{s_{i}} a_{i}\left(s_{i}-1\right) \sum_{i=1}^{n} \frac{w_{i}}{s_{i}} a_{i}} .
\end{aligned}
$$

Next we provide (design) consistent network estimators for $K_{s}$ and $\lambda_{s}$ under the AffSCR:

$$
\widehat{K}_{s 2}=\frac{\sum_{i=1}^{n} \frac{w_{i}}{a_{i}} a_{i}\left(a_{i}-1\right) \delta_{i}}{\sum_{i=1}^{n} \frac{w_{i}}{a_{i}} a_{i}\left(s_{i}-1\right) \delta_{i}}=\frac{\sum_{i=1}^{n} w_{i}\left(a_{i}-1\right) \delta_{i}}{\sum_{i=1}^{n} w_{i}\left(s_{i}-1\right) \delta_{i}},
$$

where the summand in the numerator is set $=0$, if $a_{i}=0 ; \delta_{i}$ is an indicator variable and is set $=1$, if the $i$-th sampled individual is affected and set $=0$ otherwise. In equ. 5 , each observation $i$ has a network weight of $1 / a_{i}$, because the network size for the sibship reported by sampled individual $i$ in accordance with the AffSCR is $a_{i}$. An implicit assumption required for $\hat{K}_{s 2}$ to be a consistent estimator is that for each sibship with an affected sibling each living affected sibling has a positive probability of being sampled in the survey. An estimator of SRR ratio $\lambda_{s}$ is:

$$
\hat{\lambda}_{s 2}=\widehat{K}_{s 2} / \hat{p}_{a 2}=\frac{\sum_{i=1}^{n} w_{i}\left(a_{i}-1\right) \delta_{i} \sum_{i=1}^{n} w_{i}}{\sum_{i=1}^{n} w_{i}\left(s_{i}-1\right) \delta_{i} \sum_{i=1}^{n} w_{i} \delta_{i}},
$$

where

$$
\hat{p}_{a 2}=\frac{\sum_{i=1}^{n} \frac{w_{i}}{a_{i}} a_{i} \delta_{i}}{\sum_{i=1}^{n} w_{i}}=\frac{\sum_{i=1}^{n} w_{i} \delta_{i}}{\sum_{i=1}^{n} w_{i}} .
$$

It is noteworthy that $\hat{p}_{a 2}$ reduces to the conventional sampleweighted prevalence estimator where each selected individual reports only about their own disease status. In the Appendix, we provide (design) consistent network estimators of $K_{s}$ and $\lambda_{s}$ under analogous counting rules to AllSCR and AffSCR for disease statuses of both living and deceased siblings in reported sibships.

\section{Variance Estimation of $S R R$ and $S R R$ Ratio}

Here we describe the estimators of $K_{s}$ and $\lambda_{s}$ and their variance estimators when the observations are obtained from a stratified multistage cluster sample design like the sample design of the NHIS. Let the population of individuals to be surveyed be partitioned into a set of primary sampling units (PSUs) and the PSUs be divided into $L$ sampling strata. For household surveys, the PSUs are usually geographically defined, e.g. by counties, and the strata are defined by demographic characteristics of the PSUs, e.g. the population sizes of the PSUs. At the first stage of sampling, $t_{h}$ PSUs are randomly sampled from each stratum $h(h=1, \ldots, L)$. There are usually additional stages of sampling nested within the sampled PSUs to obtain a random sample of $t_{h i}$ individuals from the $i^{\text {th }}$ sampled PSU in stratum $h$ where each sampled individual has a sample weight $w_{h i j}\left(j=1, \ldots, t_{h i}\right)$.

The estimators of $K_{s}$ and $\lambda_{s}$ in equ. 2 and 4 can be re-expressed in terms of the sample strata and PSUs:

$$
\widehat{K}_{s 1}=\frac{\sum_{h=1}^{L} \sum_{i=1}^{t_{h}} \sum_{j=1}^{t_{h i}} \frac{w_{h i j}}{s_{h i j}} a_{h i j}\left(a_{h i j}-1\right)}{\sum_{h=1}^{L} \sum_{i=1}^{t_{h}} \sum_{j=1}^{t_{h i}} \frac{w_{h i j}}{s_{h i j}} a_{h i j}\left(s_{h i j}-1\right)}
$$

and

$$
\hat{\lambda}_{s 1}=\frac{\sum_{h=1}^{L} \sum_{i=1}^{t_{h}} \sum_{j=1}^{t_{h i}} \frac{w_{h i j}}{s_{h i j}} a_{h i j}\left(a_{h i j}-1\right) \times \sum_{h=1}^{L} \sum_{i=1}^{t_{h}} \sum_{j=1}^{t_{h i}} w_{h i j}}{\sum_{h=1}^{L} \sum_{i=1}^{t_{h}} \sum_{j=1}^{t_{h i}} \frac{w_{h i j}}{s_{h i j}} a_{h i j}\left(s_{h i j}-1\right) \times \sum_{h=1}^{L} \sum_{i=1}^{t_{h}} \sum_{j=1}^{t_{h i}} \frac{w_{h i j}}{s_{h i j}} a_{h i j}} .
$$

We apply the Taylor linearization method for variance estimation, which uses a first-order Taylor expansion to approximate the variances of the estimators $\hat{K}_{s 1}$ and $\hat{\lambda}_{s 1}$ [12, pp. 23-28]. The Taylor linearization method can be implemented by first computing a quantity called the Taylor deviate for each observation, which is a measure of the influence that the observation has on the value of the estimator. The Taylor deviate is denoted below as:

$$
z_{\text {hij }}^{(K)} / w_{h i j} \text { and } z_{h i j}^{(\lambda)} / w_{h i j}
$$

for $\hat{K}_{s 1}$ and $\hat{\lambda}_{s 1}$, respectively, and can be computed by differentiating the sample-weighted estimator with respect to the sample weight of the observation [13]. Because the sample-weighted sum of the Taylor deviates is an asymptotically consistent linear approximation to the estimator for which we are obtaining a variance estimate, we can use standard survey variance formulas for estimating the variance of a weighted sum of the Taylor deviates to obtain the needed estimate of variance. The Taylor deviate and the use of differentiation with respect to the sample weights to derive the Taylor 
deviates are a convenient way to compute variance estimates for nonlinear estimators such as $\hat{K}_{s 1}$ and $\hat{\lambda}_{s 1}$.

Using the Taylor linearization method outlined above, we can approximate variances of $\hat{K}_{s 1}$ and $\hat{\lambda}_{s 1}$ as:

$$
\operatorname{Var}\left(\widehat{K}_{s 1}\right) \approx \operatorname{Var}\left[\sum_{h=1}^{L} \sum_{i=1}^{t_{h}} \sum_{j=1}^{t_{h i}} z_{h i j}^{(K)}\right]
$$

where

$$
z_{h i j}^{(K)}=w_{h i j} \widehat{K}_{s 1}\left(\begin{array}{c}
\frac{a_{h i j}\left(a_{h i j}-1\right)}{s_{h i j}} \\
\frac{a_{h i j}\left(s_{h i j}-1\right)}{s_{h i j}} \\
\frac{s_{h=1}^{t_{h}} \sum_{j=1}^{t_{h i}} \frac{w_{h i j}}{s_{h i j}} a_{h i j}\left(a_{h i j}-1\right)}{\sum_{h=1}^{L} \sum_{i=1}^{t_{h}} \sum_{j=1}^{t_{h i}} \frac{w_{h i j}}{s_{h i j}} a_{h i j}\left(s_{h i j}-1\right)}
\end{array}\right),
$$

and

$$
\operatorname{Var}\left(\hat{\lambda}_{s 1}\right) \approx \operatorname{Var}\left[\sum_{h=1}^{L} \sum_{i=1}^{t_{h}} \sum_{j=1}^{t_{h i}} z_{h i j}^{(\lambda)}\right],
$$

where

$$
z_{h i j}^{(\lambda)}=w_{h i j} \hat{\lambda}_{s 1}\left(\begin{array}{c}
\frac{a_{h i j}\left(a_{h i j}-1\right)}{s_{h i j}} \\
\left.\frac{1}{\sum_{h=1}^{L} \sum_{i=1}^{t_{h}} \sum_{j=1}^{t_{h i}} \frac{w_{h i j}}{s_{h i j}} a_{h i j}^{t_{h}} \sum_{i=1}^{t_{h i}} w_{h i j}}-1\right) \\
\frac{a_{h i j}\left(s_{h i j}-1\right)}{s_{h i j}} \\
\frac{\sum_{h=1}^{L} \sum_{i=1}^{t_{h}} \sum_{j=1}^{t_{h i}} \frac{w_{h i j}}{s_{h i j}} a_{h i j}\left(s_{h i j}-1\right)}{a_{h i j}}
\end{array}\right.
$$

Under the assumption that the sample of PSUs is approximately a stratified sample of PSUs with replacement, which is often accurate since the sampling fraction for the sampled PSUs is usually small, the variance of $\hat{K}_{s 1}$ can be estimated as:

$$
\begin{aligned}
& \widehat{\operatorname{Var}}\left(\widehat{K}_{s 1}\right) \approx \widehat{\operatorname{Var}}\left[\sum_{h=1}^{L} \sum_{i=1}^{t_{h}} \sum_{j=1}^{t_{h i}} z_{h i j}^{(K)}\right] \\
& =\sum_{h=1}^{L} \sum_{i=1}^{t_{h}}\left[z_{h i}^{(K)}-\bar{z}_{h}^{(K)}\right]^{2},
\end{aligned}
$$

where

$$
z_{h i}^{(K)}=\sum_{j=1}^{t_{h i}} z_{h i j}^{(K)} \text { and } \bar{z}_{h}^{(K)}=\frac{1}{t_{h}} \sum_{i=1}^{t_{h}} z_{h i}^{(K)} .
$$

The variance of $\hat{\lambda}_{s 1}$ can be estimated by replacing

$$
z_{h i j}^{(K)} \text { by } z_{h i j}^{(\lambda)}
$$

in the above expressions. Similar expressions can be obtained for the variance estimators of $\hat{K}_{s 2}$ and $\hat{\lambda}_{s 2}$.

\section{Results of Data Example}

\section{The 1976 NHIS Diabetes Supplement}

The network estimators of $K_{s}$ presented in this paper are illustrated with data from the 1976 NHIS and its Diabetes Supplement. The 1976 NHIS collected a US national cross-sectional random sample of civilian not institutionalized individuals of all ages, using a stratified multistage clustered probability sample design [14]. For purposes of variance estimation, the sample design is approximated by the sampling of two (pseudo)PSUs from each of the 149 (pseudo)sampling strata [15]. There is a sample weight for each of the 113,178 sampled individuals who responded to the questionnaire, which reflects the probabilities for selecting each respondent and adjustments for differential nonresponse and poststratification to US population sizes.

In the Diabetes Supplement, the following questions (paraphrased) were asked of each sampled individual who was interviewed:

Q1: Do you have diabetes?

Q2: How many living brothers and sisters do you have? (Do not count adopted, step- or half brothers and sisters.)

Q3: How many of these brothers and sisters have diabetes?

Q4: How many of your brothers and sisters are no longer living?

Q5: How many of these brothers and sisters had diabetes?

Q6: Does (did) your mother have diabetes?

Q7: Does (did) your father have diabetes?

The responses to these questions were used to obtain the diabetic status of the members of the sibship reported in the 1976 NHIS, i.e. the diabetic status of the sampled individual and their siblings and the sibship size were obtained separately for living and deceased siblings. Also, the diabetic status of the parents was used to estimate SRR and SRR ratio by parental diabetic status. 
Table 1. Number of sampled individuals in the 1976 NHIS reporting sibships with different numbers of diabetics, mortality status of diabetics, and parental diabetic status by counting rule

\begin{tabular}{|c|c|c|}
\hline Counting rule & Diabetics in the sibship & Sampled individuals \\
\hline AllSCR & $\begin{array}{l}\geq 1 \text { living diabetic } \\
\geq 2 \text { living diabetics }\end{array}$ & $\begin{array}{l}5,831 \\
1,112\end{array}$ \\
\hline AffSCR & $\begin{array}{l}\geq 1 \text { living diabetic } \\
\geq 2 \text { living diabetics }\end{array}$ & $\begin{array}{r}2,005 \\
571\end{array}$ \\
\hline AllSCR & $\begin{array}{l}\geq 1 \text { living or deceased diabetic } \\
\geq 2 \text { living or deceased diabetics }\end{array}$ & $\begin{array}{l}6,914 \\
1,533\end{array}$ \\
\hline AffSCR & $\begin{array}{l}\geq 1 \text { living or deceased diabetic } \\
\geq 2 \text { living or deceased diabetics }\end{array}$ & $\begin{array}{r}2,197 \\
712\end{array}$ \\
\hline AllSCR & $\begin{array}{l}\geq 1 \text { living diabetic, and } \geq 1 \text { parental diabetic } \\
\geq 2 \text { living diabetics, and } \geq 1 \text { parental diabetic }\end{array}$ & $\begin{array}{r}1,976 \\
536\end{array}$ \\
\hline AffSCR & $\begin{array}{l}\geq 1 \text { living or deceased diabetic, and } \geq 1 \text { parental diabetic } \\
\geq 2 \text { living or deceased diabetics, and } \geq 1 \text { parental diabetic }\end{array}$ & $\begin{array}{l}688 \\
317\end{array}$ \\
\hline
\end{tabular}

\footnotetext{
${ }^{1}$ Among the reported sibships with $\geq 2$ living siblings for reported sibships, the number involves only living diabetics; and among the reported sibships with $\geq 2$ living or deceased siblings for reported sibships, the number involves living or deceased diabetics.
}

Table 1 shows the number of individuals in the 1976 NHIS who have different diabetic statuses among their sibship plus themselves for each of the two counting rules. The rows with ' $\geq 1$ diabetics' are the sample sizes of individuals with no missing data that are utilized for the denominators of the estimators for SRR. The rows with ' $\geq 2$ diabetics' are the sample sizes that are utilized in the numerators for SRR in table 2 . We can see a reduction in sample sizes when using the AffSCR compared to the AllSCR, and an increase in sample sizes when including deceased siblings in the computation of SRR.

Table 2 presents the estimates of the prevalence, SRR, SRR ratio and their relative standard errors (RSE, i.e. the standard error for an estimate divided by the corresponding estimate) under the AffSCR and AllSCR for reporting the diabetic status of living siblings and living or deceased siblings. Also, estimates are given for sibships when at least one parent has/had diabetes. The estimates of the prevalence of diabetes were slightly higher under the AffSCR than under the AllSCR, indicating possibly more accurate reporting from affected individuals under the AffSCR than from a mix of affected and unaffected individuals under the AllSCR, assuming there is little overreport-

Sibling Recurrence Risk in Sample Surveys ing of diabetes. Also, the prevalence of diabetes was about three times larger for sibships where at least one parent was reported to have diabetes, indicating a genetic association with the development of diabetes. The estimates of SRR and SRR ratios were smaller for the AffSCR compared to the AllSCR. The smaller estimates for the AffSCR could be due to more sibships being reported with only one diabetic under the AffSCR than under the AllSCR, because the sampled individuals who are reporting their sibships' diabetic statuses are affected under this counting rule. As expected, the estimates of SRR and SRR ratios increase considerably when at least one parent was reported to have diabetes. A comparison of the RSEs shows that they are smaller for the AllSCR than for the AffSCR. This gain in efficiency is due to more individuals reporting diabetes under the AllSCR than under the AffSCR [9].

\section{Discussion}

Population-based surveys that randomly sample individuals can be used to unbiasedly ascertain the sibships of the surveyed individuals and obtain information about 
Table 2. Estimates of the prevalence, $S R R$, and $S R R$ ratio of diabetes by counting rule, mortality status of siblings, and parental diabetic status of sibships from the 1976 US NHIS

\begin{tabular}{lcll}
\hline Counting rule & $\begin{array}{l}\text { Prevalence, \% } \\
\text { (RSE, \%) }\end{array}$ & $\begin{array}{l}\text { SRR, \% } \\
\text { (RSE, \%) }\end{array}$ & $\begin{array}{l}\text { SRR ratio } \\
\text { (RSE, \%) }\end{array}$ \\
\hline $\begin{array}{l}\text { Reporting living siblings } \\
\text { AffSCR }\end{array}$ & & \\
AllSCR & $2.16(2.61)$ & $12.51(4.56)$ & $5.79(5.12)$ \\
\hline Reporting living or deceased siblings & & \\
AffSCR & 2.97 (2.43) & $13.75(4.43)$ & $5.87(4.38)$ \\
AllSCR & $2.16(1.72)$ & $15.37(3.89)$ & $7.10(3.46)$ \\
\hline
\end{tabular}

Reporting living siblings among sibships with parental diabetes

$\begin{array}{llll}\text { AffSCR } & 6.13(4.19) & 18.08(6.26) & 8.37(6.74)^{1}\end{array}$

$\begin{array}{llll}\text { AllSCR } & 6.05(2.74) & 20.93(5.20) & 10.62(5.04)^{1}\end{array}$

Reporting living siblings among sibships without parental diabetes $\begin{array}{llll}\text { AffSCR } & 1.68(2.89) & 9.50(5.99) & 4.40(6.37)^{1}\end{array}$

$\begin{array}{llll}\text { AllSCR } & 1.47(2.05) & 11.45(5.27) & 5.81(5.62)^{1}\end{array}$

Reporting living or deceased siblings among sibships with parental diabetes

$\begin{array}{llll}\text { AffSCR } & 6.51(3.86) & 19.65(5.60) & 8.39(5.74)^{2}\end{array}$

$\begin{array}{llll}\text { AllSCR } & 6.43(2.83) & 21.87(4.33) & 10.10(4.05)^{2}\end{array}$

Reporting living or deceased siblings among sibships without parental diabetes

$\begin{array}{llll}\text { AffSCR } & 1.83(2.69) & 11.11(6.40) & 4.74(6.30)^{2} \\ \text { AllSCR } & 1.64(1.96) & 12.14(6.18) & 5.61(5.89)^{2}\end{array}$

${ }^{1}$ Prevalences from the general population were used to compute SRR ratio, 2.16 and 1.97 for the AffSCR and AllSCR, respectively.

${ }^{2}$ Prevalences from the general population were used to compute SRR ratio, 2.34 and 2.16 for the AffSCR and AllSCR, respectively.

the disease status of each member of the ascertained sibships. Using appropriate counting rules that link individuals enumerated in the survey to their sibships, we show that additional data about the number of individuals who can report the ascertained sibships can be collected during the survey interviews to appropriately down-weight the disease status information of the sibships for possible multiple reporting. We provide design-based consistent estimators for SRR and SRR ratios based on two counting rules: a counting rule where either affected or unaffected individuals can report the disease statuses of themselves and their siblings, and a counting rule where only affected individuals enumerated in the survey can report about the disease statuses of themselves and their siblings. Thus, population-based surveys can provide unbiased ascertainment of sibships and disease statuses of the individu- als in the sibships that geneticists can use to accurately estimate SRR and SRR ratios to determine the extent of family aggregation of various diseases. Using the Diabetes Supplement to the 1976 NHIS, we were able to illustrate our estimation methods for calculating SRR and SRR ratios for diabetes in the US population.

Since the 1976 NHIS data did not distinguish between Type 1 and Type 2 diabetes in the questionnaire, the estimates of SRR and SRR ratios were for the population of a mixture of both types of diabetes. Household surveys could be designed to collect the diabetic status of the interviewed individuals and their relatives that is specific to the type of diabetes, although the accuracy of the reported type of diabetes should be examined with validation studies beforehand.

Using reports from the 1976 NHIS interviewed individuals about their diabetic statuses and their living siblings' diabetic statuses, we estimated the prevalence of diabetes for all ages to be about $2 \%$ and SRR ratios to be 5.8 and 7.7 depending on the counting rule (AffSCR vs. AllSCR), and we estimated higher SRR ratios of 8.4 and 10.6 for families with at least one diabetic parent. Similar sized estimates were found in a 1960s Canadian family study [16]. In that study, the sex- and age-specific SRR ratios were 14,5 , and 4 for $0-19,20-38$, and $\geq 40$ year olds, respectively, for male diabetic probands reporting about their brother's diabetic status with somewhat similar estimates for the other gender combinations. As in the 1976 NHIS data, that study appears to include both Type 1 and Type 2 diabetics. However, our estimates of SRR ratio of diabetes are higher than more current estimates obtained in the 1990s that range from 0.8 to 1.2 for families without parental diabetes, from 1.6 to 2.5 for families with one diabetic parent, and from 2.2 to 4.0 for families with two diabetic parents [17]. These SRR ratio estimates may be lower because in the 1990s the prevalence of obesity was higher in the US and other western counties than in the 1970s, resulting in higher prevalences of diabetes in the 1990s compared to the 1970s. Also, environmental causes of diabetes related to increased obesity would tend to diminish a genetic-based correlation for diabetes.

Our methods assume accurate reporting by enumerated individuals in the survey of disease statuses of siblings and of network sizes used for computing the network weights. As the example of diabetes illustrates, the estimates of the prevalence, SRR and SRR ratios differed by counting rule and also by whether or not the reported siblings were deceased. These differences could reflect the accuracy of the reported data used to produce the estimates, which may be a function of the counting rule that 
determines who is eligible to report information on the sibship. Further theoretical and empirical work is needed to develop counting rules and estimators that are robust to reporting error while maintaining good statistical efficiency. For example, hybrid counting rules that combine existing counting rules, e.g. the counting rule used for the AffSCR for reporting living and deceased siblings, may be one approach for obtaining improved estimation.

The network weights used for the AllSCR and AffSCR for living siblings were the reciprocal of the number of living siblings and the reciprocal of the number of living affected siblings, respectively. The network estimators of the prevalence and SRR can be biased if the network weights are not the reciprocal of the network sizes, e.g. if members of the sibships that can be reported by surveyed individuals live outside of the targeted population being sampled. In the case of the 1976 NHIS, which sampled from the not institutionalized US population, siblings of sampled individuals could live outside of the USA or may be institutionalized, then the network weights will be too small for these reported sibships. This error results in an overestimation of the prevalence of the disease if sibships with affected individuals are prone to the error and may over- or underestimate SRR depending on how the error relates to the affected status and size of the reported sibships. Another consideration when estimating SRR is underdiagnosis of the disease. In the case of diabetes, the Second National Health and Nutrition Examination Survey (NHANES II) conducted between 1976 and 1980 has reported that about $50 \%$ of diabetes among $20-74$ year olds is underdiagnosed [18]. Such an underdiagnosis will bias downward the estimated prevalence and SRR of diabetes from population and family studies that do not do a comprehensive medical determination of diabetes among the enumerated individuals and their siblings.

In this paper, estimators based on essentially two counting rules are presented: the AllSCR where any enumerated individual can report about the diabetic status of their sibship, and the AffSCR where only affected individuals can report about the diabetic status of their sibship. A natural question is how one should choose a counting rule. One consideration that has been discussed in the context of the diabetes example is the difference between the counting rules in the reporting accuracy of the sibship's disease status and of the network size for computing the network weights. In order to address these considerations, pilot studies should be done to validate the reported data under each counting rule by comparing the reports to medical and other record information. A second consideration is the magnitude of the sampling

Sibling Recurrence Risk in Sample Surveys error between the estimators that use each counting rule. For estimating disease prevalences under the assumption of simple random sampling of selection units, it is often but not always the case that the counting rule that results in more reported disease events will have a smaller sampling error. This was observed for estimating SRR and SRR ratio in our diabetes example under the complex sample design of the NHIS. However, other distributional characteristics such as how clustered the sampling units are within the networks will affect the magnitude of the sampling error [9]. Further analytical and empirical work is needed for comparing SRR and SRR ratio estimators for specific diseases under different counting rules in order to choose the counting rule that minimizes both bias (from reporting error) and sampling error. It should also be noted that when designing a survey other considerations involving survey operational costs can affect the choice of the counting rule, e.g. to what extent the counting rule can lengthen the average interview time for a sampled household.

Beyond providing the statistical methods for producing an approximately unbiased estimation of SRR from a national survey, this paper illustrates for diabetes that the data needed to compute the estimates can be collected in a national survey. As mentioned in the introduction, to our knowledge the 1976 NHIS is the only publicly available national survey data set that has data about disease and mortality status of sibships to allow for the estimation of family aggregation such as SRR using network estimation. There are other national surveys that have collected family histories of diseases and that for a small amount of additional effort could have collected further data to apply our network estimators of SRR. For example, NHANES II, a household medical examination survey conducted between 1976 and 1980, collected data from interviewed individuals, 12-74 years old, on the diagnosis of diabetes among their siblings and themselves and the total number of living siblings, but it did not determine how many of the living siblings were also 12-74 years old [19], information needed to compute the network weights. Another example, the 1987 NHIS collected data about the diagnosis of cancer (and the specific type of cancer) of the interviewed individuals who were 18 years and older and of their siblings, but the survey did not determine the number of living siblings (18 years and older), data needed to compute the network weights [20]. These examples along with the 1976 NHIS show that the current ongoing NHISs and NHANESs can collect the necessary information about family histories of specific diseases and family structures needed to obtain network estimates of SRR. 
When designing a questionnaire to collect data in a survey to compute network estimates for SRR, it is important to consider the information needed by the counting rules. For example, if the AffSCR is used, then if it is initially determined that the interviewed individual is unaffected, no further questions about their sibship need to be asked. Similarly for the AllSCR, if the interviewed individual reports that their sibship is of size 1 or has no affected siblings, no further questions need to be asked because the individual's sibship will not contribute to the estimation of SRR. Therefore with an appropriate ordering of the questions in a personal interview or a self-administered questionnaire along with appropriate skip patterns, there can be a significant reduction in the length of the interview or time for filling out the self-administered questionnaire, thereby reducing interviewing costs and respondent burden.

We recommend that future population-based household surveys consider family relationship network sampling to collect disease status data of siblings and other family relatives similar to the 1976 NHIS Diabetes Supplement to be able to use approximately unbiased network estimators of SRR and SRR ratios for defined target populations.

\section{Appendix}

Estimation That Includes Deceased Siblings

Two estimators of $K_{s}$, using the AllSCR and AffSCR in which the affected status of both living and deceased siblings are reported, are given by:

$$
\widehat{K}_{s 1}^{*}=\frac{\sum_{i=1}^{n} \frac{w_{i}}{s_{i}} a_{i}^{*}\left(a_{i}^{*}-1\right)}{\sum_{i=1}^{n} \frac{w_{i}}{s_{i}} a_{i}^{*}\left(s_{i}^{*}-1\right)}
$$

and

$$
\widehat{K}_{s 2}^{*}=\frac{\sum_{i=1}^{n} w_{i}\left[I_{i} \frac{\delta_{i} a_{i}^{*}\left(a_{i}^{*}-1\right)}{a_{i}}+\left(1-I_{i}\right) \frac{a_{i}^{*}\left(a_{i}^{*}-1\right)}{s_{i}}\right]}{\sum_{i=1}^{n} w_{i}\left[I_{i} \frac{\delta_{i} a_{i}^{*}\left(s_{i}^{*}-1\right)}{a_{i}}+\left(1-I_{i}\right) \frac{a_{i}^{*}\left(s_{i}^{*}-1\right)}{s_{i}}\right]},
$$

respectively, where $a_{i}^{*}$ are the number of affected siblings who are alive or deceased, $s_{i}^{*}$ are the sibship sizes for the living and deceased siblings for the sibship reported by the $i$-th sampled individual, $a_{i}$ are the number of affected individuals among the living siblings, and $I_{i}$ is an indicator variable that is 1 when $a_{i} \neq 0$, and 0 when $a_{i}=0$. The purpose of $I_{i}$ in $\hat{K}_{s 2}^{*}$ is to permit the use of a hybrid counting rule where the AffSCR is used for enumerated individuals that report $a_{i} \neq 0$ and the AllSCR is used for enumerated individuals that report $a_{i}=0$. If we had only used the AffSCR rule for all enumerated individuals, then the estimator would be biased because sibships where all affected siblings are deceased would not be counted in $\hat{K}_{s 2}^{*}$. Even though the counting rule for $\hat{K}_{s 2}^{*}$ is a hybrid of the AllSCR and AffSCR, for simplicity we refer to this counting rule as the AffSCR.

Since our definition of $K_{s}$ includes reports of siblings who are alive or deceased, we estimate $p_{a}$ using the AllSCR to obtain:

$$
\hat{p}_{1}^{*}=\frac{\sum_{i=1}^{n} \frac{w_{i}}{s_{i}} a_{i}^{*}}{\sum_{i=1}^{n} \frac{w_{i}}{s_{i}} s_{i}^{*}}
$$

and using the (hybrid) AffSCR to obtain:

$$
\hat{p}_{2}^{*}=\frac{\sum_{i=1}^{n} w_{i} \delta_{i}\left[I_{i} \frac{a_{i}^{*}}{a_{i}}+\left(1-I_{i}\right) \frac{a_{i}^{*}}{s_{i}}\right]}{\sum_{i=1}^{n} \frac{w_{i}}{s_{i}} s_{i}^{*}} .
$$

The estimators for $\lambda$ for each of the counting rules are:

$$
\hat{\lambda}_{s 1}^{*}=\frac{\sum_{i=1}^{n} \frac{w_{i}}{s_{i}} a_{i}^{*}\left(a_{i}^{*}-1\right)}{\sum_{i=1}^{n} \frac{w_{i}}{s_{i}} a_{i}^{*}\left(s_{i}^{*}-1\right)} \times \frac{\sum_{i=1}^{n} \frac{w_{i}}{s_{i}} s_{i}^{*}}{\sum_{i=1}^{n} \frac{w_{i}}{s_{i}} a_{i}^{*}}
$$

and

$$
\begin{aligned}
\hat{\lambda}_{s 2}^{*}=\frac{\sum_{i=1}^{n} w_{i}\left[I_{i} \frac{\delta_{i} a_{i}^{*}\left(a_{i}^{*}-1\right)}{a_{i}}+\left(1-I_{i}\right) \frac{a_{i}^{*}\left(a_{i}^{*}-1\right)}{s_{i}}\right]}{\sum_{i=1}^{n} w_{i}\left[I_{i} \frac{\delta_{i} a_{i}^{*}\left(s_{i}^{*}-1\right)}{a_{i}}+\left(1-I_{i}\right) \frac{a_{i}^{*}\left(s_{i}^{*}-1\right)}{s_{i}}\right]} \\
\times \frac{\sum_{i=1}^{n} \frac{w_{i}}{s_{i}} s_{i}^{*}}{\sum_{i=1}^{n} \delta_{i} w_{i}\left[I_{i} \frac{a_{i}^{*}}{a_{i}}+\left(1-I_{i}\right) \frac{a_{i}^{*}}{s_{i}}\right]} .
\end{aligned}
$$

The Taylor linearization variance estimators for $\hat{p}_{1}^{*} \hat{p}_{2}^{*}, \hat{K}_{s 1}^{*}, \hat{K}_{s 2}^{*}, \hat{\lambda}_{s 1}^{*}$, and $\hat{\lambda}_{s 2}^{*}$ are obtained in the same fashion as described in the Variance Estimation of SRR and SRR Ratio section for estimators for living siblings only.

\section{Acknowledgements}

We thank the two reviewers for their thoughtful comments and suggested changes. 


\section{References}

$>1$ Penrose LS: The genetical background of common diseases. Acta Genet Stat Med 1953; 4:257-265.

$>2$ Risch $\mathrm{N}$ : Linkage strategies for genetically complex traits. I. Multilocus models. Am J Hum Genet 1990;46:222-228.

3 Risch N: Linkage strategies for genetically complex traits. II. The power of affected relative pairs. Am J Hum Genet 1990;46:229-241.

$\checkmark 4$ Risch N: Linkage strategies for genetically complex traits. III. The effect of marker polymorphism on analysis of affected relative pairs. Am J Hum Genet 1990;46:242-253.

5 Guo SW: Inflation of sibling recurrence-risk ratio, due to ascertainment bias and/or overreporting. Am J Hum Genet 1998;63:252258.

6 Olson JM, Cordell JM: Ascertainment bias in the estimation of sibling genetic risk parameters. Genet Epidemiol 2000;18:217-235.

7 Zou G, Zhao H: The estimation of sibling genetic risk parameters revisited. Genet Epidemiol 2004;26:286-293.
8 Sutton R: Referring patients for a medical genetics consultation and genetic counseling. Adv Otorhinolaryngol 2011;70:25-27.

9 Sirken MG: Network sampling; in Encyclopedia of Biostatistics. New York, Wiley, 1998, pp 2977-2986.

10 Bensen JT, Liese AD, Rushing JT, Province M, Folsom AR, Rich SS, Higgins M: Accuracy of proband reported family history: the NHLBI Family Heart Study (FHS). Genet Epidemiol 1999; 17:141-150.

11 Sirken MG, Graubard BI, McDaniel M: National network surveys of diabetes; in Proceedings of the American Statistical Association of the Section on Survey Research Methods. American Statistical Association, 1978, pp 631-635.

12 Korn EL, Graubard BI: Analysis of Health Surveys. New York, Wiley, 1999.

13 Shah BV: Comment on 'linearization variance estimators for survey data' by A. Demnati and J.N.K. Rao. Surv Methodol 2004;30: 29.

14 Black ER: Current estimates from the Health Interview Survey; United States - 1976. Vital Health Stat 10 1977:1-80.
15 National Center for Health Statistics: Variance estimation for the 1973-1984 NHIS public use person data, 2009. http://www.cdc. gov/nchs/data/nhis/7384var.pdf.

16 Simpson NE: Diabetes in families of diabetics. Can Med Assoc J 1968;98:427-432.

17 Weijnen CF, Rich SS, Meigs JB, Krolewski AS, Warram JH: Risk of diabetes in siblings of index cases with Type 2 diabetes: implications for genetic studies. Diabet Med 2002;19:4150.

18 Harris MI, Hadden WC, Knowler WC, Bennett $\mathrm{PH}$ : Prevalence of diabetes and impaired glucose tolerance and plasma glucose levels in U.S. population aged 20-74 years. Diabetes 1987;36:523-534.

19 McDowell A, Engel A, Massey JT, Mauer K: Plan and operation of the Second National Health and Nutrition Examination Survey, 1976-1980. Vital Health Stat 1 1981:1-144.

20 Schoenborn CA, Marano M: Current estimates from the National Health Interview Survey: United States, 1987. Vital Health Stat 10 1988:1-233. 